\title{
Archaea prevalence in inflamed pulp tissues
}

\author{
MAGDALENA EFENBERGER ${ }^{l}$, JUSTYNA AGIER ${ }^{l}$, ELŻBIETA PAWŁOWSKA ${ }^{2}$, \\ EWA BRZEZIŃSKA-BEASZCZYK ${ }^{1}$
}

${ }^{1}$ Department of Experimental Immunology, Medical University of Lodz, Lodz, Poland

${ }^{2}$ Department of Orthodontics, Medical University of Lodz, Lodz, Poland

\begin{abstract}
Archaea have been detected in several ecological niches of the human body such as the large intestine, skin, vagina as well as the oral cavity. At present, archaea are recognized as nonpathogenic microorganisms. However, some data indicate that they may be involved in the etiopathogenesis of several diseases, including intestinal diseases as well as oral diseases: periodontitis, peri-implantitis and endodontitis. In this study, on the basis of $16 \mathrm{~S}$ rRNA gene sequence analysis, we examined whether archaea might be present in inflamed pulp tissues and contribute to the development of endodontic infection. In comparison, we also determined selected bacterial species associated with endodontitis. We detected archaea in $85 \%$ of infected endodontic samples. In addition, Prevotella intermedia, Porphyromonas gingivalis, Tannerella forsythia and Treponema denticola were present in inflamed pulp tissue samples and Treponema denticola occurred with the highest frequency (70\%). Further analysis revealed the presence of methanogenic archaea in analyzed samples. Direct sequencing of archaeal $16 S$ rRNA gene PCR products indicated the occurrence of methanogenic archaea in inflamed pulp tissues; phylogenetically most similar were Methanobrevibacter oralis and Methanobrevibacter smithii. Therefore, our results show that methanogenic archaea are present in inflamed pulp tissues and may participate in the development of endodontic infection.
\end{abstract}

Key words: archaea, inflammation, Methanobrevibacter oralis, endodontic infection, inflamed pulp tissues.

(Centr Eur J Immunol 2015; 40 (2): 194-200)

\section{Introduction}

Archaea are microorganisms classified as a third, separate domain of life. They are phylogenetically distant from bacteria and even more closely related to eucarya [1]. Although archaeal overall cell organization is quite similar to that in bacteria, archaea have some specific features. Their cell wall is devoid of peptidoglycan and their membrane lipids contain ether linkages - far more stable than bacterial ester linkages. In addition archaea have some unique external structures like archaella, pili, hami and cannulae $[2,3]$. The best studied group of archaea is methanogens strictly anaerobic microorganisms which synthesize methane using simple inorganic compounds like $\mathrm{CO}_{2}$ and $\mathrm{H}_{2}$ in the process of methanogenesis [4]. Originally, archaea were thought to be a primitive form of life, living only in extreme environments like hot springs and salt lakes but recent studies have indicated that they are even more ecologically widespread.

Although archaea were firstly detected in natural ecosystems, emerging data indicate that they might be closely associated with human microbiome [5, 6]. High amounts of methanogens have been detected in the human gastroin- testinal tract, especially in the large intestine [7, 8]. Moreover, methanogens have been found among the microbial flora of the human vagina [9]. Recently, Probst et al. [10] has demonstrated that ammonia oxidizing archaea may be a part of human skin microbiome. Taking into consideration that human microbiome plays an important role in many physiological processes, one can assume that archaea also participate in the maintenance of homeostasis in several ecological niches of the human body. Even though archaea are now recognized as nonpathogenic microorganisms and as a natural component of human microbiota, some data reveal that archaea may participate in the development of various diseases. Nevertheless, the potential role of archaea in etiopathogenesis of several diseases is still poorly understood. At present, there is no clear evidence that archaea could be pathogenic microorganisms and none of them has been described as a pathogenic agent. Recently, however, some associations between the presence of methanogens and the occurrence of intestinal diseases such as colon cancer [11], inflammatory bowel disease [12], irritable bowel disease [13], and even obesity [14] have been reported. 
Several studies have shown that archaea could be involved in some oral diseases, nonetheless the available data are very limited. Archaea were detected in subgingival plaque samples [15-17] and tongue scrapings [18] from patients with periodontitis. It should be also stressed that a significant association between the severity of periodontal disease and the prevalence of archaea was observed [15, 17]. In addition, Faveri et al. [19] indicated an increased prevalence of archaea in peri-implantitis sites. Some data revealed that archaea are present within inflamed dental pulp tissue and thus might be associated with endodontic infection [2023]. Therefore, the purpose of this study was to analyze the prevalence and diversity of archaea in infected endodontic samples from patients with primary endodontic infection in comparison with selected bacterial species involved in endodontitis such as Prevotella intermedia, Porphyromonas gingivalis, Tannerella forsythia, and Treponema denticola.

\section{Material and methods}

\section{Patient selection}

A total of 20 patients over the age of 18 years were enrolled in this study. The patients underwent root canal treatment in the Dental Institute at the Medical University of Lodz. The study was approved by the Bioethics Committee of the Medical University of Łódź, Poland (approval number RNN/280/14/KB) and informed consent was obtained from all patients in accordance with the Declaration of Helsinki. Complete medical and dental histories were taken from all patients participating in this study. The patients were otherwise healthy and those who had received antibiotic treatment during the previous 3 months were excluded from this investigation.

\section{Sample collection}

One endodontic sample per patient was collected. The tooth was isolated with a rubber dam. The crown and the surrounding area were disinfected with $30 \%$ hydrogen peroxide for $30 \mathrm{~s}$ and next with $5.25 \%$ sodium hypochlorite for additional $30 \mathrm{~s}$. In order to neutralize disinfectant agents, $5 \%$ sodium thiosulfate was used. An access cavity was prepared with sterile high-speed diamond burs under irrigation with sterile saline and it was disinfected in the same way. The samples were collected with four sterile paper points which were placed in the root canal. Finally, the paper points were placed in a sterile tube containing $1 \mathrm{ml}$ of transport fluid (PBS), immediately frozen and stored at $-80^{\circ} \mathrm{C}$ until further analysis.

\section{Extraction of total genomic DNA}

Deep-frozen endodontic samples were thawed and dispersed by constant stirring for $30 \mathrm{~min}$. Paper points were removed and samples were centrifuged at $12000 \mathrm{rpm}$ for
$10 \mathrm{~min}$. After centrifugation the supernatant was discarded and the pellet containing microbial cells was suspended in $100 \mu \mathrm{l}$ of Tris- $\mathrm{HCl}$ buffer $(\mathrm{pH}=8.5)$. The microbial DNA was extracted from inflamed pulp tissues using Genomic Mini kit (A\&A Biotechnology, Gdynia, Poland) according to the manufacturer's protocol. The cells were lysed using lysing solution and with proteinase K. DNA was selectively adsorbed onto silica matrix of the spin column and purified from the contaminants. Finally, total extracted genomic DNA was suspended in a solution containing $100 \mu \mathrm{l}$ of TE buffer (Tris-EDTA, $\mathrm{pH}=7.4)$. DNA concentration $(A 260)$ and the purity (A260/A280) were measured using a Picodrop Microliter Spectrophotometer (Picodrop Limited, UK). DNA samples were immediately frozen at $-80^{\circ} \mathrm{C}$ and stored for further analysis.

\section{PCR amplification}

DNA extracted from infected pulp tissues was amplified using 2720 ThermalCycler (Life Technologies, USA). The abundance of archaea and some selected bacteria (Prevotella intermedia, Porphyromonas gingivalis, Tannerella forsythia, Treponema denticola) was analyzed using oligonucleotide specific primers. Primer sequences used for detection of 16S rRNA gene from total archaea and 16S rRNA gene from selected endodontic bacteria are presented in Table 1. Primer sequences and modified PCR conditions for archaea and bacteria detection were as described by Vianna et al. [20] and Vickerman et al. [21], respectively. The specificities and annealing temperatures of the PCR primers for archaea and bacteria detection were verified in silico using the Primer-BLAST program at NCBI (http://www.ncbi.nlm. nih.gov/BLAST/). The annealing temperature of each primer-pair was analyzed in order to avoid primer dimer interference. PCR was performed in a total volume of $25 \mu \mathrm{l}$. Each PCR mixture contained approximately $5 \mathrm{ng}$ of template DNA, $10 \mu \mathrm{M}$ of forward and reverse primers and DreamTaq Green PCR Master Mix (Thermo Scientific, USA) containing DreamTaq DNA polymerase, $0.4 \mathrm{mM}$ dNTPs and $4 \mathrm{mM}$ $\mathrm{MgCl}_{2}$. The DNA samples were preheated at $95^{\circ} \mathrm{C}$ for $3 \mathrm{~min}$ and amplified for 40 cycles under the following conditions: denaturation at $95^{\circ} \mathrm{C}$ for $30 \mathrm{~s}$, annealing at the primer specific annealing temperature for $30 \mathrm{~s}$, elongation at $72^{\circ} \mathrm{C}$ for $30 \mathrm{~s}$ followed by final elongation at $72^{\circ} \mathrm{C}$ for $7 \mathrm{~min}$. After PCR amplification the products were separated by electrophoresis in a $2 \%$ agarose gel with ethidium bromide in $1 \times \mathrm{TBE}$ buffer (90 mM Tris-borate, 2 mM EDTA) and visualized under UV light. Genomic DNA extracted from subgingival plaque sample, which was earlier sequenced and was found to be positive for archaea, was applied as positive PCR control in each reaction. PCR conditions and specificity of PCR primers for archaea detection were further experimentally ensured by PCR with the reference DNA extracted from Methanobrevibacter oralis DSM 7256 purchased from German Collection of Microorganisms and Cell Cultures, 
Table 1. PCR primers and conditions for microbial detection

\begin{tabular}{|c|c|c|c|c|}
\hline Microorganism & Sequence $\left(5^{\prime} \rightarrow 3^{\prime}\right)$ & $\begin{array}{l}\text { Amplicon size } \\
\text { (bp) }\end{array}$ & $\begin{array}{l}\text { Annealing } \\
\text { temp. }\left({ }^{\circ} \mathrm{C}\right)\end{array}$ & Reference \\
\hline Total archaea & $\begin{array}{l}\text { ACKGCTCAGTAACACGT } \\
\text { GTGCTCCCCCGCCAATTCCT }\end{array}$ & 793 & 56 & 20 \\
\hline Prevotella intermedia & $\begin{array}{c}\text { CGTGGACCAAAGATTCATCGGTGGA } \\
\text { CCGCTTTACTCCCCAACAAA }\end{array}$ & 260 & 57 & 21 \\
\hline Porphyromonas gingivalis & $\begin{array}{l}\text { AGGCAGCTTGCCATACTGCG } \\
\text { ACTGTTAGCAACTACCGATGT }\end{array}$ & 405 & 57 & 21 \\
\hline Tannarella forsythia & $\begin{array}{l}\text { TACAGGGGAATAAAATGAGATACG } \\
\text { ACGTCATCCCAACCTTCCTC }\end{array}$ & 746 & 57 & 21 \\
\hline Treponema denticola & $\begin{array}{l}\text { TAATACCGAATGTGCTCATTTACAT } \\
\text { TCAAAGAAGCATTCCCTCTTCTTCTTA }\end{array}$ & 316 & 57 & 21 \\
\hline
\end{tabular}

Braunschweig, Germany. DNA isolated from a subgingival plaque sample of periodontally healthy individuals which was earlier found to be negative for archaea was used as negative control. The second negative control contained ultrapure water instead of template DNA.

\section{Sequencing}

Archaeal species were identified on the basis of $16 \mathrm{~S}$ rRNA gene sequence by direct sequencing of representative archaeal PCR products. Archaeal DNA samples were amplified using PCR method and separated using electrophoresis as described above. Next, the amplified archaeal DNA samples were purified with Clean Up kit (A\&A Biotechnology, Gdynia, Poland) according to the manufacturer's protocol. PCR products were sequenced using 3130xl Genetic Analyser (Applied Biosystems, Warrington, UK) and the same forward primers as for PCR amplification were used (Table 1).

\section{Phylogenetic analysis}

The obtained archaeal 16S rRNA gene sequences were precisely analyzed using Sequence Scanner 1.0 software and incorrectly aligned positions were edited. The sequences were compared with those available in the international sequence database GenBank using the BLAST server at the National Centre for Biotechnology Information (NCBI) (http://www.ncbi.nlm.nih.gov/BLAST/). Multiple sequence alignment was performed using ClustalW2 program. The phylogenetic tree reconstruction was made with MEGA 6.0 software package by the neighbor-joining method and the bootstrap values were generated.

\section{Results}

\section{Prevalence of bacteria and archaea in inflamed pulp tissue samples}

We first estimated, using PCR assay and gel electrophoresis, bacterial species present in inflamed pulp tissue samples. We noticed that $50 \%$ of infected pulp tissues were positive for single bacterial species, the other constituted multispecies samples. Two species of bacteria were detected in $30 \%$ of samples, three bacterial species were found in $15 \%$ of samples and only $5 \%$ of infected pulp tissues were positive for all bacterial species. The most prevalent was Treponema denticola detected in $70 \%$ of endodontic samples. Tannerella forsythia was found in $45 \%$ of samples and Porphyromonas gingivalis was present in $40 \%$ of samples. Prevotella intermedia occurred with the lowest frequency, inasmuch as only $20 \%$ of samples were positive. Moreover, on the basis of $16 \mathrm{~S}$ rRNA gene sequence analysis we investigated the prevalence of members of the domain Archaea in endodontic samples. We documented that these microorganisms were present in $85 \%$ of analyzed inflamed pulp tissue samples (Table 2).

\section{Identification of archaea}

In order to identify archaea in infected root canals we conducted direct sequencing of selected representative archaeal 16S rRNA gene PCR products. The obtained sequences were compared with those available in GenBank database. Figure 1 depicts the phylogenetic affiliation of $16 \mathrm{~S}$ rRNA gene sequence (using endodontic sample 3 as a representative sequence) in comparison with representative members of five archaeal phylums: Euryarchaeota, Crenarchaeota, Korarchaeota, Nanoarchaeota, and Thaumarchaeota. We stated that archaea prevalent in infected root canals undoubtedly belonged to the group of methanogens (phylum: Euryarchaeota). The sequences obtained from endodontic samples were very similar to methanogens belonging to the Methanobrevibacter species, but also showed a sequence similarity of 91-95\% to the Methanobacterium species. All sequences were closely related to Methanobrevibacter oralis strain ZR and shared 98-99\% sequence identity. The sequenced amplicons also revealed high similarity values to Methanobrevibacter smithii strain PS (97-99\%), Methanobrevibacter thaueri strain CW (97-98\%), Methanobrevibacter millerae strain ZA-10 (96-98\%). The sequences obtained from samples 3, 
Table 2. The prevalence of bacteria and archaea in endodontic samples

\begin{tabular}{|c|c|c|c|c|c|}
\hline $\begin{array}{c}\text { No. of endodontic } \\
\text { sample }\end{array}$ & $\begin{array}{c}\text { Prevotella } \\
\text { intermedia }\end{array}$ & $\begin{array}{l}\text { Porphyromonas } \\
\text { gingivalis }\end{array}$ & $\begin{array}{c}\text { Tannarella } \\
\text { forsythia }\end{array}$ & $\begin{array}{c}\text { Treponema } \\
\text { denticola }\end{array}$ & Archaea \\
\hline 1 & - & + & + & + & + \\
\hline 2 & + & - & + & + & + \\
\hline 3 & - & - & + & - & + \\
\hline 4 & - & + & + & + & + \\
\hline 5 & + & - & - & + & + \\
\hline 6 & + & - & + & - & + \\
\hline 7 & - & - & - & + & + \\
\hline 8 & + & + & + & + & + \\
\hline 9 & - & + & - & + & + \\
\hline 10 & - & + & - & + & + \\
\hline 11 & - & - & - & + & + \\
\hline 12 & - & - & - & + & + \\
\hline 13 & - & - & - & + & + \\
\hline 14 & - & - & + & - & + \\
\hline 15 & - & + & - & - & - \\
\hline 16 & - & - & + & + & + \\
\hline 17 & - & + & + & - & + \\
\hline 18 & - & + & - & - & - \\
\hline 19 & - & - & - & + & + \\
\hline 20 & - & - & - & + & - \\
\hline
\end{tabular}

6, 9 and 10 showed equal similarity to Methanobrevibacter smithii strain PS as well as to Methanobrevibacter oralis strain ZR (99\%). Moreover, amplicons from samples 3 and 9 revealed Methanobrevibacter gottschalkii strain $\mathrm{HO}$ as a very close relative and shared $98 \%$ identity.

\section{Discussion}

Pulpitis may be triggered by several external factors, including trauma from accident or bruxism, improper cavity preparation during dental treatment or contaminated restorative materials. All these factors facilitate microbial invasion and thereby infection of the root canal system. Without any doubt, the most common cause of pulpitis is indirect bacterial infection. Bacteria may enter the root canal mainly via caries, but also via dental anomalies, lateral canals or damaged cementum. The most frequent bacteria in the infected root canal belong to the phylums Firmicutes, Proteobacteria, Spirochaetes, Bacteroidetes, and Actinobacteria [24, 25]. However, some data indicate differences in microbiota of primary and secondary endodontic infections [26, 27]. Primary endodontic infections are dominated by Fusobacterium sp., Porphyromonas sp., Enterococcus sp., Prevotella sp., Treponema sp., Tan- nerella sp., Parvimonas sp., Lactobacillus sp. and Propionibacterium sp. [24-27]. In our study we examined the prevalence of selected species of bacteria in primary endodontic infection. We demonstrated that Prevotella intermedia, Porphyromonas gingivalis, Tannerella forsythia, and Treponema denticola occurred in inflamed pulp tissues and the most prevalent was Treponema denticola. Thus, our studies are consistent with the current state of knowledge.

Although bacteria are the main causative agent of endodontitis, some data suggest that other microorganisms, such as fungi, especially Candida albicans [28] and viruses [29], can participate in the development of pulp tissue inflammation, as well. Recently, it has been discussed whether members of the domain Archaea might be another etiological agent of endodontic infection. Nowadays, however, the available data are very limited. Vianna et al. [20] detected archaea in $25 \%$ of necrotic pulp tissues. Vickerman et al. [21] indicated the presence of archaea in $1 / 20$ of symptomatic and 1/14 of asymptomatic endodontic infections. Jiang et al. [23] stated that archaea occurred in $38 \%$ of root canal samples from teeth with primary infections and in $17 \%$ of cases diagnosed as secondary root infected canals. In our study we demonstrated the pres- 


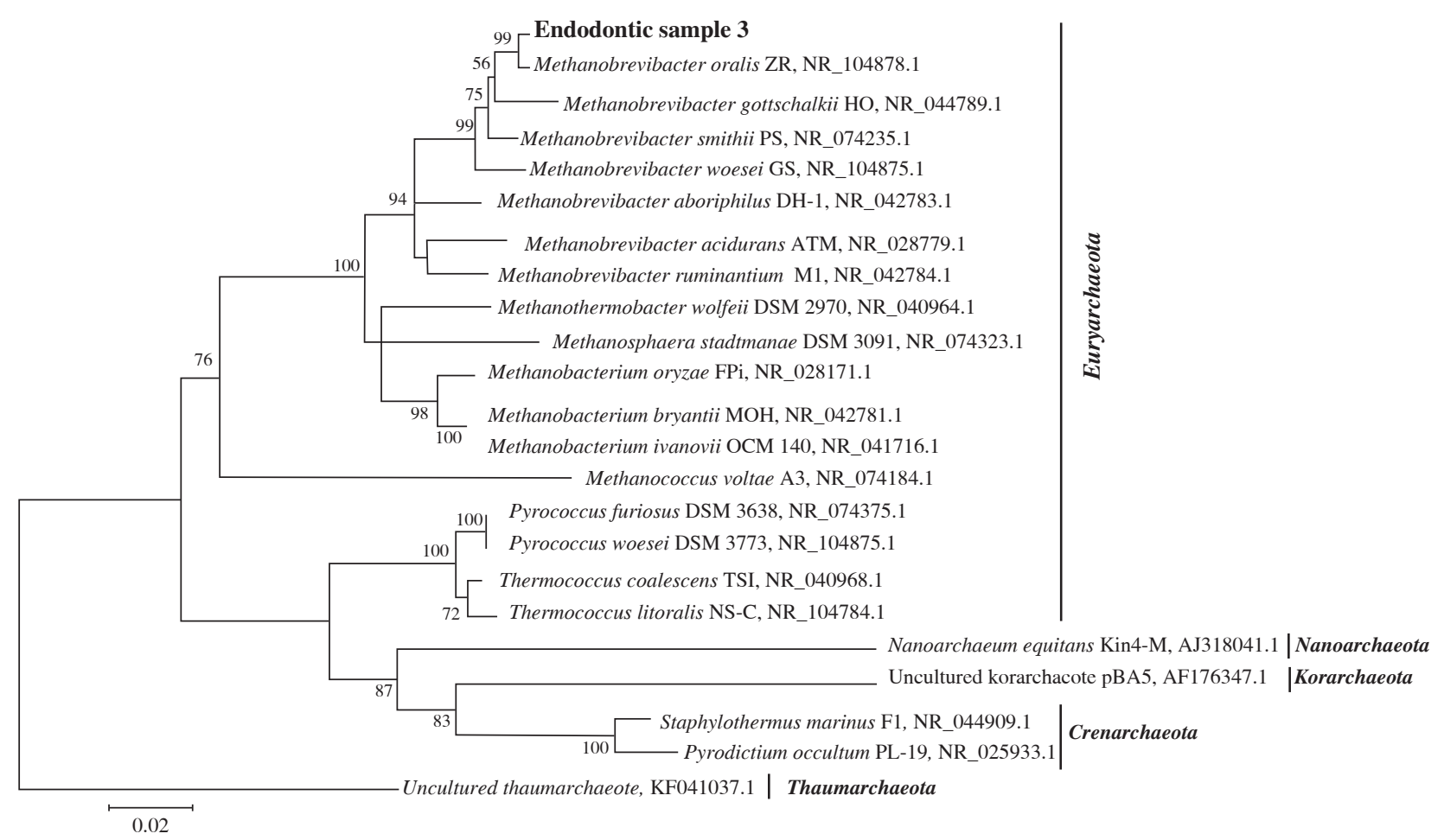

Fig. 1. Phylogenetic tree showing the position of archaeal 16S rRNA gene sequences identified in infected endodontic samples in comparison with representative species of five phylums: Euryarchaeota, Nanoarchaeota, Korarchaeota, Crenarchaeota and Thaumarchaeota. Phylogenetic tree was constructed for representative endodontic sample 3 . The scale bar is 0.02 substitutions per nucleotide. The tree was made using neighbor-joining method with bootstrap analysis. The bootstrap values lower than 50 are not shown

ence of archaea in $85 \%$ of infected pulp tissues using the same primers like Vianna et al. [20]. We suppose that such a huge discrepancy between results may be due to rules of patient selection and criteria for patient inclusion. It is also possible that microbiological variations between different populations exist. Baumgartner et al. [25] demonstrated that differences in bacteria detected in endodontic infections might be underlined by geographical locations.

Previously, it has been shown that archaea associated with endodontic infection belong to the group of methanogens [20-23]. Moreover, there are no data indicating the presence of other groups of archaea in infected pulp tissues. Thus, it seems that methanogens may be the only archaea involved in dental root disease. Direct sequencing of PCR products based on 16S rRNA gene sequence found the domination of Methanobrevibacter oralis-like phylotype in infected pulp tissues [20, 21]. The results indicated the presence of methanogens in inflamed pulp tissues. Comparative analysis of archaeal 16S rRNA gene sequence demonstrated that oral inhabitant Methanobrevibacter oralis can occur in inflamed pulp tissues. The obtained 16S rRNA gene sequences were almost identical to this archaeon and shared $98-99 \%$ identity. However, $4 / 9$ of obtained sequences also shared $99 \%$ similarity to
Methanobrevibacter smithii, thus this archaeon may be also detected in endodontic samples. Moreover, we cannot exclude the occurrence of other methanogens like Methanobrevibacter thaueri, Methanobrevibacter millerae and Methanobrevibacter gottschalkii.

The question whether archaea could be potential human pathogens remains still open. Previous observations [20-23] as well as our results clearly demonstrated that infected pulp tissue is colonized not only by several bacterial species but also by archaea, especially methanogens. This leads to the hypothesis that archaea in cooperation with bacteria may take part in the course of pulp tissue inflammation. However, the coexistence between bacteria and archaea is not clear. It was suggested that this cooperation depends on syntrophic interactions between bacteria and archaea connected with hydrogen transfer [15, 22]. Recently, it has been shown that there is a possible competition for $\mathrm{H}_{2}$ between archaea, sulfate-reducing bacteria and acetogenic bacteria Treponema sp. It was indicated that these three hydrogenotrophic microbial groups may function as alternative syntrophic partners of secondary fermenting periodontal pathogens $[15,17]$. In addition, these interactions may be very important because Treponema denticola is a well-known oral pathogen and sulfate reducing bacteria are able to synthesize hydrogen sulfide toxic for tissues. 
Table 3. Identification of archaea in endodontic samples on the basis of 16S rRNA gene sequence

\begin{tabular}{|c|c|c|c|c|c|c|c|c|c|}
\hline \multirow[t]{3}{*}{ Archaeal strains } & \multicolumn{9}{|c|}{ No. of endodontic sample } \\
\hline & 1 & 3 & 4 & 6 & 8 & 9 & 10 & 16 & 17 \\
\hline & \multicolumn{9}{|c|}{ Sequence identity [\%] } \\
\hline Methanobrevibacter oralis $Z R$ & 99 & 99 & 98 & 99 & 99 & 99 & 99 & 99 & 98 \\
\hline Methanobrevibacter smithii PS & 98 & 99 & 97 & 99 & 98 & 99 & 99 & 98 & 97 \\
\hline Methanobrevibacter thaueri $\mathrm{CW}$ & 98 & 98 & 97 & 98 & 98 & 98 & 97 & 97 & 97 \\
\hline Methenobrevibacter millerae $\mathrm{ZA}-10$ & 98 & 98 & 96 & 98 & 98 & 98 & 98 & 97 & 97 \\
\hline Methanobrevibacter gottschalkii HO & 97 & 98 & 96 & 96 & 97 & 98 & 96 & 96 & 96 \\
\hline Methanobrevibacter woesei GS & 97 & 97 & 96 & 97 & 97 & 97 & 96 & 96 & 96 \\
\hline Methanobrevibacter arboriphilus DH-1 $16 \mathrm{~S}$ & 94 & 95 & 95 & 94 & 95 & 96 & 95 & 95 & 94 \\
\hline Methanobrevibacter acididurans ATM & 94 & 95 & 95 & 94 & 94 & 96 & 96 & 95 & 94 \\
\hline Methanobrevibacter boviskoreani $\mathrm{JH} 1$ & 94 & 94 & 95 & 96 & 95 & 96 & 96 & 94 & 95 \\
\hline Methanobrevibacter ruminantium $M 1$ & 94 & 95 & 95 & 94 & 94 & 95 & 94 & 94 & 95 \\
\hline Methanobrevibacter wolinii $\mathrm{SH}$ & 94 & 94 & 94 & 95 & 94 & 95 & 93 & 94 & 94 \\
\hline Methanobrevibacter cuticularis RFM-1 & 94 & 95 & 93 & 94 & 95 & 93 & 92 & 93 & 93 \\
\hline Methanobrevibacter olleyae KM1H5-1P & 94 & 95 & 94 & 94 & 95 & 95 & 96 & 94 & 95 \\
\hline Methanobrevibacter curvatus RFM-2 & 93 & 94 & 92 & 93 & 92 & 93 & 93 & 93 & 93 \\
\hline Methanobacterium flexile $G H$ & 92 & 94 & 93 & 92 & 94 & 95 & 93 & 94 & 92 \\
\hline Methanobrevibacter filiformis RFM-3 & 92 & 93 & 92 & 93 & 92 & 93 & 93 & 92 & 92 \\
\hline
\end{tabular}

The results with identity lower than $92 \%$ are not shown.

Nowadays, nothing is known about archaeal virulence factors. Moreover, there is no knowing whether archaea invasion can induce the response of the immune system. Yamabe et al. [30] documented that Japanese patients with severe periodontitis had IgG antibodies against Methanobrevibacter oralis. It was also shown that the chaperonin subunits of Methanobrevibacter oralis were recognized by the host immune system of periodontitis patients [31, 32]. Taking into account that archaea are not only the important members of human microbiome, but also can be involved in the etiopathogenesis of some diseases it seems to be of great importance to find out whether archaeal components stimulate immune response.

The authors declare no conflict of interest.

This work was supported by the Medical University of Łódź (Grant No. 502-03/6-164-01/502-64-082).

\section{References}

1. Woese CR, Fox GE (1977): Phylogenetic structure of the prokaryotic domain: the primary kingdoms. PNAS 74: 50885090.

2. Jarrell KF, Ding Y, Nair DB, Siu S (2013): Surface appendages of archaea: structure, function, genetics and assembly. Life 3: 86-117.
3. Albers SV, Meyer BH (2011): The archaeal cell envelope. Nat Rev Microbiol 9: 414-426.

4. Schäfer G, Engelhard M, Müller V (1999): Bioenergetics of the Archaea. Microbiol Mol Biol Rev 63: 570-620.

5. Efenberger M, Wódz K, Brzezińska-Błaszczyk E (2014): Archeony - istotny składnik mikrobiomu człowieka. Przegl Lek 6: 346-351.

6. Dridi B, Raoult D, Drancourt M (2011): Archaea as emerging organisms in complex human microbiomes. Anaerobe 17: 56-63.

7. Dridi B, Henry M, El Khéchine A, et al. (2009): High prevalence of Methanobrevibacter smithii and Methanosphaera stadtmanae detected in the human gut using an improved DNA detection protocol. PLoS One 4: e7063.

8. Eckburg PB, Bik EM, Bernstein CN, et al. (2005): Diversity of the human intestinal microbial flora. Science 308: 16351638.

9. Belay N, Mukhopadhyay B, Conway de Macario E, et al. (1990): Methanogenic bacteria in human vaginal samples. J Clin Microbiol 28: 1666-1668.

10. Probst AJ, Auerbach AK, Moissl-Eichinger C (2013): Archaea on human skin. PLoS One 8: e65388.

11. Mira-Pascual L, Cabrera-Rubio R, Ocon S, et al. (2014): Microbial mucosal colonic shifts associated with the development of colorectal cancer reveal the presence of different bacterial and archaeal biomarkers. J Gastroenterol.

12. Scanlan PD, Shanahan F, Marchesi JR (2008): Human methanogen diversity and incidence in healthy and diseased colonic groups using $m c r A$ gene analysis. BMC Microbiol 8: 79. 
13. Lee KN, Lee OY, Koh DH, et al. (2013): Association between symptoms of irritable bowel syndrome and methane and hydrogen on lactulose breath test. J Korean Med Sci 28: 901-907.

14. Zhang H, DiBaise JK, Zuccolo A, et al. (2009): Human gut microbiota in obesity and after gastric bypass. PNAS 106 : 2365-2370.

15. Lepp PW, Brinig MM, Ouverney CC, et al. (2004): Methanogenic Archaea and human periodontal disease. PNAS 101: 6176-6181.

16. Li CL, Liu DL, Jiang YT, et al. (2009): Prevalence and molecular diversity of Archaea in subgingival pockets of periodontitis patients. Oral Microbiol Immunol 24: 343-346.

17. Vianna ME, Holtgraewe S, Seyfarth I, et al. (2008): Quantitative analysis of three hydrogenotrophic microbial groups, methanogenic archaea, sulfate-reducing bacteria, and acetogenic bacteria, within plaque biofilms associated with human periodontal disease. J Bacteriol 190: 3779-3785.

18. Göhler A, Hetzer A, Holtfreter B, et al. (2014): Quantitative molecular detection of putative periodontal pathogens in clinically healthy and periodontally diseased subjects. PLoS One 9: e99244.

19. Faveri M, Gonçalves LF, Feres M, et al. (2011): Prevalence and microbiological diversity of Archaea in peri-implantitis subjects by $16 \mathrm{~S}$ ribosomal RNA clonal analysis. J Periodontal Res 46: 338-344.

20. Vianna ME, Conrads G, Gomes BP, Horz HP (2006): Identification and quantification of archaea involved in primary endodontic infections. J Clin Microbiol 44: 1274-1282.

21. Vickerman MM, Brossard KA, Funk DB, et al. (2007): Phylogenetic analysis of bacterial and archaeal species in symptomatic and asymptomatic endodontic infections. J Med Microbiol 56: 110-8.

22.Vianna ME, Conrads G, Gomes BP, Horz HP (2009): T-RFLP-based $m c r A$ gene analysis of methanogenic archaea in association with oral infections and evidence of a novel Methanobrevibacter phylotype. Oral Microbiol Immunol 24: 417-422.

23. Jiang YT, Xia WW, Li CL, et al. (2009): Preliminary study of the presence and association of bacteria and archaea in teeth with apical periodontitis. Int Endod J 42: 1096-1103.

24. Saito D, Leonardo Rde T, Rodrigues JL, et al. (2006): Identification of bacteria in endodontic infections by sequence analysis of $16 \mathrm{~S}$ rDNA clone libraries. J Med Microbiol 55: 101-107.

25. Baumgartner JC, Siqueira JF Jr, Xia T, Róças IN (2004): Geographical differences in bacteria detected in endodontic infections using polymerase chain reaction. J Endod 30: 141-144.

26. Hong BY, Lee TK, Lim SM, et al. (2013): Microbial analysis in primary and persistent endodontic infections by using pyrosequencing. J Endod 39: 1136-1140.

27. Tennert C, Fuhrmann M, Wittmer A, et al. (2014): New bacterial composition in primary and persistent/secondary endodontic infections with respect to clinical and radiographic findings. J Endod 40: 670-677.

28. Baumgartner JC, Watts CM, Xia T (2000): Occurrence of Candida albicans in infections of endodontic origin. J Endod 26: 695-698.

29. Li H, Chen V, Chen Y, et al. (2009): Herpesviruses in endodontic pathoses: association of Epstein-Barr virus with irreversible pulpitis and apical periodontitis. J Endod 35: 23-29.

30. Yamabe K, Maeda H, Kokeguchi S, et al. (2008): Distribution of Archaea in Japanese patients with periodontitis and hu- moral immune response to the components. FEMS Microbiol Lett 287: 69-75.

31. Yamabe K, Maeda H, Kokeguchi S, et al. (2010): Antigenic group II chaperonin in Methanobrevibacter oralis may cross-react with human chaperonin CCT. Mol Oral Microbiol 25: 112-122.

32. Hirai K, Maeda H, Omori K, et al. (2013): Serum antibody response to group II chaperonin from Methanobrevibacter oralis and human chaperonin CCT. Pathog Dis 68: 12-19. 